Voix et Images

voixetimages

\title{
Le corps accidenté chez Hélène Monette et Élise Turcotte
}

L'Amérique des lieux clos

The Uneven Body in the Work of Hélène Monette and Élise

Turcotte

\section{An america of closed spaces}

El cuerpo accidentado en Hélène Monette y Élise Turcotte

La américa de los lugares cerrados

\section{Marie Parent}

Volume 37, numéro 1 (109), automne 2011

URI : https://id.erudit.org/iderudit/1006466ar

DOI : https://doi.org/10.7202/1006466ar

Aller au sommaire du numéro

Éditeur(s)

Université du Québec à Montréal

ISSN

0318-9201 (imprimé)

1705-933X (numérique)

Découvrir la revue

Citer cet article

Parent, M. (2011). Le corps accidenté chez Hélène Monette et Élise Turcotte :

l'Amérique des lieux clos. Voix et Images, 37(1), 115-128.

https://doi.org/10.7202/1006466ar
Résumé de l'article

Cet article propose d'interroger une fois de plus le concept d'américanité, cette fois-ci à partir de deux textes d'écrivaines québécoises contemporaines, Unless d'Hélène Monette et Pourquoi faire une maison avec ses morts d'Élise Turcotte. À travers la figure du corps " accidenté » sera mise en évidence la relation de réciprocité établie dans ces textes entre corps et territoire : la représentation du corps blessé, souffrant est indissociable de l'expérience d’une Amérique torturée, hantée par le sentiment de sa fin imminente. Corps et continent, pris l'un pour l'autre, trahissent ici le même malaise, et fondent d'une manière particulière la difficulté à habiter le monde. 


\section{LE CORPS ACCIDENTÉ CHEZ HÉLÈNE MONETTE ET ÉLISE TURCOTTE \\ L'Amérique des lieux clos ${ }^{1}$}

$+++$

MARIE PARENT

Université du Québec à Montréal

Loin d'avoir envie de partir, j'éprouve plutôt le besoin de rester;

et non content de rester je songe même à m'enfoncer, à descendre davantage

dans le lieu que j'occupe. Traverser l'Amérique, alors, non pas d'est en ouest ni du nord au sud,

mais vers le fond, le dedans ${ }^{2}$.

Dans Intérieurs du Nouveau Monde, Pierre Nepveu renouvelle l'approche théorique du concept d'américanité, en prenant pour postulat l'habitation fondamentalement problématique de l'Amérique. Nepveu redéfinit l'américanité non seulement comme expérience du territoire mais comme rencontre de l'altérité sous toutes ses formes, le sujet se heurtant violemment à ce qu'il ne connaît pas du monde et de lui-même. Il identifie dans les littératures des Amériques depuis leur fondation « une autre manière d'être dans le Nouveau Monde ${ }^{3}$ ", caractérisée par un mouvement de "retour sur soi ${ }^{4}$ » $s^{\prime}$ inscrivant à rebours du mythe des grands espaces et visant à affronter cette nature sauvage enfouie en soi.

C'est dans cette optique que seront analysées les œuvres de deux écrivaines québécoises contemporaines, Unless ${ }^{5} \mathrm{~d}^{\prime}$ Hélène Monette et Pourquoi faire une maison avec ses morts ${ }^{6} \mathrm{~d}^{\prime}$ Élise Turcotte. Bien que très différents par le ton et la forme, ces textes, qui n'ont d'ailleurs jamais été étudiés dans la perspective de l'américanité, soulèvent une même question : est-il possible d'habiter l'Amérique? Perdues au cœur d'une grande ville nord-américaine, les narratrices tentent de tracer les limites d'un lieu habitable, limites qui ne cessent de reculer, mettant en péril l'intégrité du sujet. Le corps «accidenté», plus qu'une image thématique, servira ici de figure structurante,

$$
+++
$$

1 Cet article a été écrit dans le cadre d'un projet de recherche soutenu par le CRSH (Conseil de recherches en sciences humaines du Canada) et le FQRSC (Fonds québécois de recherche sur la société et la culture). 2 René Lapierre, Écrire l'Amérique, Montréal, Les Herbes rouges, coll. «Essais», 1995, p. 10. 3 Pierre Nepveu, Intérieurs du Nouveau Monde. Essais sur les littératures du Québec et des Amériques, Montréal, Boréal, coll. «Papiers collés», 1998, p. 27. 4 Ibid., p. 31.5 Hélène Monette, Unless, Montréal, Boréal, 1995, 187 p. Désormais, les références à ce roman seront indiquées par le sigle $U$, suivi du folio, et placées entre parenthèses dans le texte. 6 Élise Turcotte, Pourquoi faire une maison avec ses morts, Montréal, Leméac, 2007, 124 p. Désormais, les références à ce recueil seront indiquées par le sigle $P$, suivi du folio, et placées entre parenthèses dans le texte. 
permettant de mettre en évidence la relation de réciprocité établie entre corps et territoire. On emploie l'adjectif « accidenté» pour désigner autant un terrain qui présente des inégalités, des obstacles, qu'un individu victime d'une chute, d'un choc, d'une collision. Ainsi, dans ces textes, la représentation du corps blessé, souffrant, est indissociable de l'expérience d'un territoire mouvementé, forçant le sujet à retraiter au plus creux de lui-même et à mesurer sa propre étrangeté.

Dans un premier temps, il s'agira de montrer comment la ville, la maison et le corps constituent une enfilade de lieux clos, conduisant vers l'angoisse de la mort et de la destruction. Tant le roman de Monette que les récits de Turcotte font entendre la voix de «ceux qui ne crient plus et descendent les rues par en dedans » $(U, 9)$, esquissant le portrait d'une Amérique intérieure et secrète, hantée par le sentiment de sa fin imminente. Dans un deuxième temps sera exposée cette Amérique des catastrophes, l'exact envers de celle des scénarios hollywoodiens. Les cataclysmes auxquels s'attachent Monette et Turcotte, qu'ils se situent à l'échelle mondiale ou locale, sont racontés depuis des lieux intimes et oubliés, lieux en marge de l'Histoire ou même des actualités médiatiques. C'est à partir de ces lieux précaires et excentrés que nous verrons comment s'écrit cette autre Amérique, exiguë, sombre et organique.

\section{DÉCLINAISON DU LIEU CLOS: CORPS, MAISON, VILLE}

Le rapport à l'espace en Amérique est marqué par une soif d'habiter culturellement le territoire (toujours plus à l'ouest, plus au nord...), cette quête s'assimilant à une épopée nationale. Le continent semble exister pour être traversé et raconté de part en part; c'est pourquoi les aventuriers et les vagabonds y font si bonne figure. Pourtant, dans les textes étudiés ici se fonde un rapport à l'espace infiniment plus ambigu que le rapport habituellement énoncé dans les romans s'inscrivant dans l'imaginaire de la Frontière. S'y établit un réseau de lieux clos (corps, maison, ville), dont l'interaction révèle la tentative d'habiter le continent et ses résultats ambivalents.

Le roman de Monette donne la parole à trois sœurs, parmi lesquelles Unless, personnage principal sur lequel portera surtout notre analyse, tandis que le recueil de Turcotte présente sept récits, attribués implicitement à la même narratrice, mère de deux enfants. Unless et la narratrice de Pourquoi faire une maison avec ses morts partagent un passé marqué par la mort et le drame. La quête principale de ces deux personnages deviendra de trouver un refuge, un endroit pour se mettre à l'abri des tempêtes qui secouent leur existence. En ce sens, nous chercherons à montrer que leur parcours réactualise l'expérience fondatrice de la vie en Amérique, c'est-à-dire de l'installation sur un territoire et de son habitation ${ }^{7}$. Cette expérience se trouve au

7 L'établissement d'un parallèle entre la colonisation du continent et la tentative du sujet de «trouver sa place » dans l'Amérique contemporaine peut sembler grossier, mais rappelons que notre réflexion se situe strictement sur les plans imaginaire et symbolique. À ce sujet, la définition de l'habitation que propose Mircea Eliade est éclairante: «[...] remarquons qu'habiter un territoire, c'est-à-dire s'installer, bâtir une demeure, implique toujours une décision vitale qui engage l'existence de la communauté tout entière. Se "situer" dans un paysage, l'organiser, 
cœur de toutes les entreprises de colonisation, comme l'explique le géographe sinoaméricain Yi-Fu Tuan:

Pensons à la manière dont un pays est colonisé. Au départ, tout est sauvage ; l'espace est indifférencié. On aménage une clairière dans la forêt et on construit quelques maisons. Immédiatement, une différenciation se produit; d'un côté il y a la forêt à l'état sauvage et de l'autre un monde petit, vulnérable, construit par l'homme. [...] Avec l'extension continue des clairières, la forêt finit par disparaître. Un paysage entier est humanisé. [...] Les limites d'une colonie ne sont plus clairement visibles. Elles ne sont plus mises en relief par les frontières évidentes de la nature sauvage. Aussi l'intégrité du lieu doit-elle être entretenue de manière rituelle ${ }^{8}$. (Nous soulignons.)

Le processus de la colonisation implique qu'il faut déterminer clairement un dedans et un dehors, établir une différenciation entre soi et tout ce qui est conçu comme autre. Les textes à l'étude ici permettent justement de montrer comment ces limites sont sensibles, poreuses. L'espace propre doit constamment être redéfini, mais le sujet ne possède pas les ressources symboliques qui lui permettraient d'assurer l'intégrité du chez-soi. Son corps même deviendra le lieu d'une osmose avec le monde, le dehors apparaissant comme le prolongement du dedans et vice-versa. Dans son échec à tracer le périmètre de son refuge, le sujet devra affronter l'espace indifférencié du continent.

\section{L'ODYSSÉE D'UNLESS}

Un jour, un ami d'Unless l'a rebaptisée Ulyssette. À l'instar du héros de L'Odyssée, son parcours prend la forme d'une interminable quête du chez-soi, mais contrairement à Ulysse qui finira par rejoindre Ithaque, Unless erre sans fin, puisqu'elle ne connaît pas sa destination finale. La jeune femme habite Monryal, où elle compte bien s'installer: "Pour commencer, il faut emménager ici. Déménager, partir d'ailleurs, d’un autre quartier populaire. Il faut décider quand émigrer et où. [...] On sait où aller, où revenir, où et qui être. On est chez soi. On reste longtemps.» $(U, 10)$ Mais trouver un endroit où faire son nid n'est pas une mince affaire. «La violence régulière» $(U, 161)$ de la ville et des contacts humains menace le corps d'Unless de toutes parts. Messagère à vélo, elle traverse la ville d'un bout à l'autre à toute vitesse, affrontant les voitures, la glace, le froid, la souffrance ordinaire des gens qu'elle ne côtoie que quelques

$$
+++
$$

l'habiter - sont des actions qui présupposent un choix existentiel : le choix de "l'Univers" que l'on est prêt à assumer en le "créant". [...] Toute installation humaine, qu'il s'agisse de la prise de possession d'un pays tout entier ou de l'élévation d'une simple demeure, répète donc la cosmogonie.» (L'auteur souligne.) Mircea Eliade, "Architecture sacrée et symbolisme», Constantin Tacou (dir.), Les symboles du lieu. L'habitation de l'homme, Paris, Éditions de L'Herne, 1983, p. 69-70. 8 Yi-Fu Tuan, Espace et lieu. La perspective de l'expérience, traduit de l'anglais par Céline Perez, Gollion (Suisse), Infolio, coll. «Archigraphy Paysages», 2006 [1977], p. 167. 
secondes à la fois, quelques secondes de trop, souvent. Le genou abîmé, les muscles endoloris, à bout de souffle, elle pédale en tentant d'éviter les obstacles, sans y parvenir : les passants à l'air déprimé, une vieille connaissance qui étale son succès, un sidéen à l'agonie à qui elle livre une carte en forme de papillon. Ces rencontres brutales la laissent plus meurtrie encore que sa course dans les rues montréalaises. Son circuit, marqué par les mouvements d'entrée et de sortie, d'arrivée et de départ, apparaît comme une fuite effrénée de tous ces lieux étouffants qui constituent la ville: tours à bureaux, boutiques, hôpitaux. Sa voix porte cette urgence, l'écriture reproduisant pratiquement la pulsation cardiaque de la narratrice, le rythme affolé de ses pérégrinations.

Pourtant, quand elle finit ses journées, elle aimerait bien se poser quelque part et rêve d'un refuge, d'une "tanière, près d'un cours d'eau » $(U, 178)$. Son appartement, situé dans un quartier pauvre et délabré, fait figure d'abri temporaire: les moments de grâce vécus avec son amant alternent avec les moments de drame, quand débarque un membre de sa famille éclatée. La vaisselle et les meubles cassés, les cris et les larmes remplissent alors cette «maison électrocutée» $(U, 42)$. Le logis d'Unless sert d'escale pour les rescapés et devient le plus souvent le terrain de leur affrontement. Au contraire d'un port d'attache, il constitue plutôt un lieu de transit où chacun contemple l'ampleur de sa solitude et de son déracinement: «Le plancher, du couloir au salon, ressemblait à celui d'une gare après tous les départs. C'est ça, après. Ceux qui restent sont là. Sans domicile fixe. » $(U, 105)$ La maison évoque ici l'opposé de ce que Gaston Bachelard décrivait dans sa Poétique de l'espace, c'est-à-dire un lieu de bonheur, de rêverie, qui «maintient l'homme à travers les orages du ciel et les orages de la vie ${ }^{9}$ ". Elle est précisément le lieu où se déchaînent les orages. «Enclave» $(U, 34)$, « taudis » $(U, 105)$, elle apparaît à la fois coupée du monde et fragilisée par lui.

Écorchée par une histoire familiale traumatisante, Unless mène un combat incessant pour sa survie, physique, morale, affective. Le corps, unique zone de contact entre le sujet et son environnement, est évidemment le premier touché. «Mon âme, c'est mon corps", déclare Unless d'emblée $(U, 25)$. La douleur, sous toutes ses formes, se loge dans ses os, ses muscles, ses organes. Le corps se fait lourd, portant dans sa chair les marques du passé. Mais Unless s'est résolue à partager son corps avec cette «poussière épaisse» $(U, 63)$ : «Au piège, nous étions rendus. [...] Regardant la misère tomber au fond de nous [...].» $(U, 63)$ Quand son frère se suicide, le mal qui squatte le corps d'Unless explose et se répand dans le texte, se retourne contre le texte:

Voici le chaos. Le chaos et notre guerre. Des sandwiches faits de peau, d'organes et de cheveux. Le fils de ma mère. Ce bruit au milieu de la nuit. Cette vie de perte et d'enfants. Rentrer dedans. Le fils de son père. Voilà l'absence révélée, le cratère du monde et la fosse. Voici notre amour fragmenté. Les filles et le père. (U, 59; l'auteure souligne.)

Le corps à bout de nerfs se dresse là, entre ces phrases tronquées, cette voix qui sursaute. «Sandwiches faits de peau, d'organes et de cheveux»: les images dérivées du

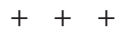

9 Gaston Bachelard, La poétique de l'espace, Paris, Presses universitaires de France, coll. «Quadrige», 1992 [1957], p. 26. 
corps non seulement contaminent le texte, mais le menacent, puisqu'en permettant à l'écriture d'approcher l'indicible, elles la rapprochent aussi du mutisme. Entre chaque énoncé prend place un silence, une fissure, qui devient une manière de parler depuis l'écart, depuis la blessure. Mais trouée par ces silences qui deviennent, dans ce contexte, plus significatifs que les mots, la voix énonciative risque de s'éteindre.

C'est rouler à vélo qui, tout en permettant au personnage de survivre à ces épreuves, rendra possible la continuation du texte. Surtout, rouler instaure une nouvelle façon de s'approprier la ville, non pas en allant d'un endroit à l'autre, mais en filant entre les lieux, là où le sujet dispose de l'espace dont il a besoin pour respirer:

\begin{abstract}
[Ā vélo], on glisse dans le réel, mais comme toujours à la lisière, mieux, à côté, dans un nulle part bien précis. [...] À pied, ça nous fait autrement palpiter, ça écrase, les immeubles sont vraiment des immeubles, terribles, brillants, et les êtres humains, des frôlements, des inquiétudes [...]. (U, 103-104)
\end{abstract}

La vitesse semble abstraire l'espace, créer une brèche à travers le temps, plongeant le personnage dans un non-lieu synonyme de bien-être. Cette solution intermédiaire serait pour Unless la seule manière possible d'être au monde. «Habiter la ville, [...] c'est faire escale, affirme Pierre Ouellet, [...] la loi des villes étant qu'à tout moment il faut circuler, circuler, circuler ${ }^{10} \ldots$.. La menace de l'enfermement est ainsi tenue à distance, les frontières du corps, du logis, de la ville rompues par l'ivresse du mouvement. «Nulle part» devient la patrie d'Unless, là vers où elle doit retourner.

\title{
SUR LA FRONTIËRE
}

L'idée d'une circulation salutaire habite aussi les récits du recueil Pourquoi faire une maison avec ses morts d'Élise Turcotte, mais, comme chez Monette, le mouvement ne prend pas la forme d'une résolution totale. La narratrice évolue dans une grande ville située au bord d'un "fleuve infesté de maladies» $(P, 9-10)$. Obsédée par la mort dès son plus jeune âge, elle ne voit dans les paysages urbains qu'un vaste cimetière: «Dans le métro, à la pharmacie, sur la rue déambulaient des morts en sursis.» $(P, 11)$ Les morts violentes se multiplient autour d'elle, la laissant coupable, hagarde, insomniaque. Pour se soulager, elle parcourt la ville, sans destination. Mais bien qu'elle circule, la narratrice n'arrive pas à s'échapper. En effet, «la ville produit un effet aussi diaboliquement carcéral que [notre] propre corps ${ }^{11}$ ", affirme Jean-François Chassay. Que ce soit dans les maisons, les wagons des métros ou les magasins à grande surface, la ville se trame entre une enfilade de lieux clos, qui nourrissent sa claustrophobie. Et puis, il y a les rues, les ruelles, les parcs, à travers lesquels la narratrice peut tracer son propre chemin, inscrire sa fugace présence dans un circuit dont elle est le seul

10 Pierre Ouellet, «Ethos urbain. La pente, l'enclave et autres lieux de perdition», Globe. Revue internationale d'études québécoises, vol. 5, nº 1, 2002, p. 27-28. 11 Jean-François Chassay, Dérives de la fin. Sciences, corps \& villes, Montréal, Le Quartanier, coll. «Erres essais», 2008, p. 132. 
témoin. Mais il s'agit d'une lutte perdue d'avance: «Nous nous trouvions déjà, sans le savoir, sur la pente conduisant à un cruel enfermement.» $(P, 33)$ Le personnage de Pourquoi faire une maison avec ses morts prétend que son errance « [l'] éloigne de la mort et $\left[l^{\prime}\right]$ en rapproche à la fois » $(P, 15)$. En effet, l'errance soulage la narratrice du poids de soi, du sentiment de sa propre fin, alors qu'elle l'expose au poids des autres, du monde, à un sentiment de la fin plus diffus mais plus englobant. Sa perception des gens, des décors, de la vie s'affine et s'altère à la fois, dotant son corps d'un caractère de perméabilité. Elle raconte un épisode vécu à dix-sept ans dans un centre commercial :

\begin{abstract}
J'ai fermé les yeux et me suis souvenue d'un rêve où l'on me coupait les jambes. Sirène du centre commercial, j'avais peut-être abdiqué sur quelques détails après tout. [...] J'ai alors senti ma seconde vie commencer. Une vie entre mon corps vivant et le corps d'un être mort. Je flottais entre les boutiques, naviguais parmi les vieillards et les désœuvrés. Peut-être n'étais-je pas tout à fait née? Ou peut-être qu'une défunte habitait mon corps parce qu'elle n'était pas tout à fait prête à partir. J'étais hantée. $(P, 16)$
\end{abstract}

La jeune fille réalise qu'elle se tient sur le seuil de la mort, prête à basculer. Pourtant, quelque chose la retient dans la vie; elle est donc condamnée à occuper cet entredeux, son corps étant lui-même habité par une entité étrangère, peut-être « une femme enterrée vivante» $(P, 17)$. Bouger, marcher dans la ville permet d'inscrire cet état transitoire dans la durée, établit pour la narratrice un espace de survie, entre l'angoisse et la paix, tandis que s'arrêter voudrait dire être avalée par le monde définitivement. En ce sens, la maison représente pour la narratrice un endroit asphyxiant, mortifère:

\begin{abstract}
Les maisons elles-mêmes étaient des tombeaux pour les morts-vivants. [...] Les odeurs de nourriture cuisant dans les fourneaux, les lumières des salons où j'imaginais l'activité quotidienne des gens, leur solitude le soir venu, leurs voix sortant des fenêtres, les piaillements d'enfants, les animaux en boule au pied des lits, tout ça, si humain, si mortel. $(P, 11)$
\end{abstract}

Le corps et la maison portent dans leur matérialité même l'intuition de leur propre finitude. La narratrice et ses enfants habitent une maison meublée par le deuil et le chagrin, où ils se trouvent de plus en plus isolés. L'espace domestique se fait menaçant, comme s'il avait aspiré les maladies du sujet, les laissant flotter dans l'air, pour mieux l'empoisonner tranquillement: "Nous n'en savions rien encore, bien sûr, nous nous croyions heureux, tandis que notre corps, et la maison, et ensuite l'oiseau de la maison savaient. Quelque chose allait arriver.» $(P, 34)$ «La maison elle-même semblait claudiquer » $(P, 41)$, remarque la narratrice, illustrant le rapport de symétrie entre le sujet et son habitat. Elle attend que «la blessure voi[e] le jour» $(P, 27)$, comme si celle-ci allait être éjectée des murs d'un instant à l'autre. Une étrangeté radicale prend progressivement possession de ses membres, de son territoire; la narratrice voudrait s'en libérer en trouant son corps, mais elle sait que ce serait vain. L'espace intime apparaît impossible à délimiter, entièrement occupé par la terreur et la mort. 
La narration elle-même est peu à peu envahie par des éléments extérieurs, faits divers, événements mondiaux, commentaires anthropologiques, récits de deuils. Étrangement, ce sont ces voix autres, traversant celle de la narratrice, qui la sortent de l'impasse. L'image de la maison subit un déplacement important : le texte lui-même fait figure de lieu hospitalier pour les âmes perdues, mortes ou vivantes. Plus on progresse dans le recueil, plus les récits sont morcelés, plusieurs passages apparaissant sans rapport avec l'histoire du personnage principal, comme si le texte absorbait les chocs à la place du sujet ou, à tout le moins, avec lui. Ainsi se tisse un nouveau rapport au réel, sur le mode de la fragilité et de l'éclatement, puisqu'un inconnu peut vous envoyer un coup de poing au ventre à n'importe quel moment et qu'il faut apprendre à vivre avec cette «ombre installée à demeure» $(P, 83)$.

«Je suis juste à la frontière» $(U, 64)$, affirme Unless. Sur la frontière entre la vie et la mort, entre la raison et la folie, entre soi et l'Autre. De la même manière, la narratrice de Pourquoi faire une maison avec ses morts, après avoir vécu « au bord du précipice» $(P, 37)$, devient le «batelier qui aide à passer la frontière» $(P, 60)$. Dans leur quête du chez-soi, les deux femmes se trouvent confinées à cet espace liminaire, le seul qu'elles puissent véritablement investir. Si l'imaginaire de l'Amérique a souvent été représenté par la figure de la frontière (sur le mode de l'exploration et de la conquête ${ }^{12}$ ), ces textes nous permettent de penser l'américanité plutôt comme l'expérience d'un seuil intérieur, expérience reconduite de diverses manières et pour diverses raisons depuis l'époque de la découverte: entre autres parce que la colonisation du Nouveau Monde se fonde essentiellement sur la destruction des peuples amérindiens et de leurs cultures, si bien que l'homme blanc, en cherchant à délimiter son territoire, à repousser les frontières du monde sauvage, ne rencontre que sa propre barbarie. On peut aussi y voir le signe d'une américanité proprement québécoise : le Québec, enclave francophone au nord-est du continent, entretient une relation ambiguë avec la frontière (celle-ci devant être dépassée et renforcée), qui permet de mesurer l'ampleur de la menace que représente l'Autre, toujours trop près de soi ${ }^{13}$. Dans ce contexte, la nature sauvage et la civilisation, le dehors et le dedans, l'étranger et le familier montrent une étonnante réversibilité, dans leur opposition même.

Voilà ce que découvrent les narratrices de Turcotte et de Monette et qui constitue la source de leur effroi : la menace est toute proche, impossible à repousser. Leur parcours à travers cette Amérique des lieux clos ne correspond pas seulement à une exploration strictement introspective, mais permet aussi de tracer la cartographie intérieure d'un continent irrespirable, paralysé par la peur. Le corps accidenté exhibé ici est bien celui de l'Amérique. Turcotte, en écrivant ses récits dans le contexte post11 septembre, donne la parole à un sujet sous le choc, les yeux fixés sur la plaie béante

$$
++
$$

12 Voir à ce sujet Frederick Jackson Turner, The Frontier in American History, New York, H. Holt and Company, 1920, 375 p. Dès 1894, l'historien Turner fait de la conquête de l'Ouest et de l'atteinte de la côte du Pacifique le vecteur principal de l'identité états-unienne. Sur l'étude de ce thème dans l'imaginaire québécois, voir Maurice Lemire, Le mythe de l'Amérique dans l'imaginaire "canadien", Québec, Nota bene, 2003, 236 p. 13 Voir à ce sujet Simon Harel, «L'Amérique ossuaire», Le voleur de parcours, Montréal, XYZ éditeur, 1999 [1989], p. 177230. 
que représente Ground Zero. Chez Monette, dont le roman date d'une dizaine d'années plus tôt, on sent déjà ce sentiment d'insécurité généralisé qui plane au-dessus d'un continent incapable de soigner les âmes douloureuses qui s'y multiplient.

\title{
L'AMÉRIQUE DES CATASTROPHES
}

L'idée de l'Amérique suscite des images, des mots bien précis: mégapole, autoroute, vitesse, violence, voiture, prospérité, individualisme, jeunesse, déchéance. Pourtant, dans Écrire l'Amérique, René Lapierre nous met en garde contre ces clichés à partir desquels on mesure «le degré d'américanité» d'une œuvre:

\begin{abstract}
On croit parfois - on croit souvent, devrais-je dire - que l'américanité du romancier ou du poète québécois se reconnaît à des systèmes thématiques, aux emprunts référentiels qu'ils pratiquent et qui mettent plus ou moins de l'avant certaines images, certaine vision de l'Amérique contemporaine (qui est alors le plus souvent synonyme, dans un sociotexte mimétiquement très compact, des USA). Mais l'américanité n'est pas seulement un décor, et ne se mesure pas en fonction des clichés (la ville, la violence, etc.) auxquels nous renvoient spontanément la télévision et les actualités ${ }^{14}$.
\end{abstract}

Si la critique n'a généralement pas lu Unless et Pourquoi faire une maison avec ses morts en fonction du sociotexte nord-américain dont parle ici Lapierre, on ne peut pas dire que ces œuvres le rejettent, au contraire. Les figures typiques de l'imaginaire américain sont plutôt réinvesties depuis le corps du sujet, menacé de toutes parts. Ville, violence, luttes individuelles, éclatement social et décadence ne sont donc jamais abordés de manière frontale, mais plutôt du revers, comme s'il s'agissait de retourner sur elle-même l'Amérique telle qu'elle est dépeinte par le cinéma hollywoodien, à la fois catastrophique et rédemptrice. Ainsi, lorsqu'elle raconte son histoire, Unless voudrait en tirer des images d'une tristesse douce et poétique, loin de l'agitation et des scénarios explosifs:

Ce que je vois? Un film qui ne serait pas américain. Premier plan: un tas de beautés naturelles, de l'eau, un sapin, un vieux. Deuxième plan: un train, de la neige, des bottes anciennes, quelqu'un qui pleure. [...] Séquence suivante : la violence normale dans une ville ordinaire. Que des horreurs subliminales. Des psychopathes et des fusils. Un gun, un ostie de gun épeurant qui brille. [...] Quatrième essai : rien à faire avec ce film, c'est déjà infesté de partout; vus d'avion, des morceaux choisis d'une grosse ville écœurante, sur fond de musique techno et sirènes de flics. [...] La fin du film est parasitée. Le XxI ${ }^{e}$ siècle, l'Amérique, tout ce qui se branche et me fout à l'eau. $(U, 73-74)$

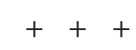

14 René Lapierre, Écrire l'Amérique, p. 11. 
Les textes de Monette et de Turcotte pourraient être parasités par cette violence ostentatoire. Pourtant ils lui résistent: ce n'est pas la violence en elle-même qui est spectaculaire ici, mais sa force de frappe. Les œuvres nous donnent accès à cette sourde douleur qui résonne encore le long des membres, bien après le moment d'un impact.

\section{LA COMMUNAUTÉ DES ACCIDENTÉS}

Tant chez Monette que chez Turcotte est exposé un éclatement familial, social, spirituel à travers lequel le sujet se trouve plus isolé, plus démuni que jamais. Unless et la narratrice de Pourquoi faire une maison avec ses morts tenteront pourtant de recréer une forme de cohésion entre les individus, en devenant chacune à leur façon le centre d'une communauté d' "accidentés», des êtres marqués à tout jamais par un destin catastrophique. Évoluant toutes deux au sein d'un foyer monoparental, elles entreprendront de refonder une cellule familiale élargie, cellule qui s'avèrera aussi suffocante que rassurante. Ainsi, le rapport à l'autre se développe toujours de manière ambiguë, celui-ci représentant à la fois la menace ultime et le seul salut possible.

Unless s'occupe périodiquement de Red, sa jeune sœur délinquante, et de Chut, son frère psychotique. Ceux-ci nous laissent entrevoir une société qui ne tolère pas les manifestations de la douleur et de la folie, puisqu'il n'y a plus aucun espace réservé pour elles - le surnom "Chut» est en ce sens éloquent. Apparaît autour d'eux une constellation d'intervenants (policiers, médecins, travailleurs sociaux) qui les assomment à coup de menaces et de médicaments. Les personnages retournent alors cette violence contre eux-mêmes, s'attaquant à leur propre corps: Chut se suicide; Red s'enfonce dans la drogue et les pratiques destructrices; Milou, la sœur aînée, enchaîne les thérapies où «elle rebirthe tout le temps» $(U, 28)$, jusqu'à vouloir mourir. Cette dernière dira $\mathrm{d}^{\prime}$ ailleurs que «leurs gènes sont meurtris» $(U, 172)$. Le mal-être et la folie constituent le seul héritage qu'aient reçu ces êtres, et ils semblent condamnés à le transmettre. Dans sa tentative de résister à la "maladie» du malheur, Unless s'efforce de «retaper les meurtris » $(U, 38)$, voit s'attrouper autour d'elle les paumés, les poqués, les esseulés qui traînent sur sa route. Ceux-ci partagent la même détresse, la «violence accumulée» $(U, 90)$ qui se déverse parfois sous forme de paroles, parfois sous forme de coups. L'un tente de soigner l'autre, mais «il n'est pas si bien équipé. Il n'a que sa tristesse» $(U, 102)$. L'univers d'Unless prend ainsi la forme d'une gigantesque infirmerie:

Quand l'infirmerie a été ouverte, elle était déjà pleine. Rengaines neurasthéniques, fabuleuses hypocondries, délires secrets et pieux malaises. Ça ne pouvait pas guérir. Ça ne voulait pas mourir parce qu'il y avait, encore et partout, du malheur à semer jusqu'à en faire fleurir des éclats meurtriers parmi les cailloux. Des chagrins tout partout. Ça perdurait à désirer la tristesse, ça puait la peine, c'était malade pour toujours, un point c'est tout. $(U, 68-69)$

«C'était malade pour toujours», dit-elle en désignant le monde qui l'entoure. Le sol, ensemencé de malheur, se recouvre de ces «éclats meurtriers » qui blessent les corps, ceux-ci déversant à leur tour leur fiel dans la terre, selon un infernal cycle de la mort. 
C'est cette boucle qu'Unless tente de briser en recueillant les corps abîmés, mais ceuxci l'enchaînent peu à peu à leur désespoir. La forme du récit, en faisant entendre plusieurs voix, à la fois concurrentes et solidaires, reproduit bien cette ambivalence marquée dans le rapport à l'autre. La tension entre les voix (représentée soit par les fréquents changements de narrateur, soit par la présence du discours indirect libre) traduit celle d'une communauté toujours au bord d'éclater, témoignant d'un tissu social particulièrement affaibli. Cette communauté s'organise en fait selon un unique principe, celui de l'instinct de conservation propre à chacun : «Les Teresa d'Amérique se préoccupent surtout de sauver leur peau $[\ldots . .$. .» $(U, 49)$ La lutte pour la survie dans ce pays, c'est la loi du chacun pour soi.

La narratrice de Pourquoi faire une maison avec ses morts se retrouve elle aussi au centre d'une communauté d'êtres fragiles et dépouillés, à commencer par ses deux enfants, qu'elle tente désespérément de protéger de la peur et de la folie qui déferlent sur le monde :

Le jour où les tours s'étaient effondrées à New York, alors que tout le monde ici avait les yeux rivés sur la télévision, moi, j'emmenais ma fille en voiture pour lui faire voir que la vie continuait, dans les rues, dans les parcs, dans les cafés [...]. Je devais soigner une jeune fille qui grandissait. $(P, 81-82)$

Alors qu'elle et ses enfants absorbent malgré eux l'atmosphère toxique qui se dégage de ce «continent [...] avalé par une vague meurtrière», «les personnes les plus concernées refusaient de voir l'angoisse et la détresse» $(P, 82)$. Une distance infranchissable se creuse entre ceux qui souffrent et les autres, vaquant à leurs occupations ordinaires, comme anesthésiés. La narratrice et ses enfants, au contraire, ressentent très distinctement les échos des catastrophes les plus lointaines, la douleur entrant «par les pores de [leur] peau comme par les antennes d'un animal affolé» $(P, 76)$. Que ce soit lors de deuils collectifs, comme dans le cas du 11 septembre, ou de deuils personnels, le drame de la mort est désormais escamoté. Le sujet se découvre sans ressources devant le deuil, la société ayant évacué l'ensemble des rituels traditionnels visant à le canaliser: «On accepte si bien la mort que la dépouille d'une personne aimée peut passer directement de l'hôpital au four. Comme s'il ne s'était rien passé. Pas d'attente. Pas de sacrifice. Pas de perte. Pas de larmes. À peine des souvenirs.» $(P, 49)$ Pour pallier cette absence de lieu où vivre la souffrance, la narratrice s'invente une occupation, en devenant accompagnatrice de fin de vie. Ce ne sont pas les mourants qu'elle accompagne, mais bien ceux qui leur survivent. Elle accueille les endeuillés, écoute leurs confidences, $s^{\prime}$ emploie à «donne[r] forme aux blessures» $(P, 21)$. Elle les soulage comme elle peut, en fabriquant des autels qui ressemblent à des maisons de poupées. Ainsi, les vivants peuvent imaginer qu'à défaut de trouver un endroit pour apaiser leur chagrin, leurs défunts, eux, en posséderont un. Mais ces artifices compensatoires n'apportent malgré tout qu'une bien maigre consolation, «ce sont des symboles aussi dérisoires que des roulottes disséminées dans un paradis vague » $(P, 60-61)$. Bien que ceux qui souffrent se réunissent et tentent de résister ensemble au mal qui les submerge, la narratrice discerne dans leur désarroi les signes d'un monde qui court à la ruine: «Je sais que la fin du monde est proche parce que le récit de la mort a été coupé.» $(P, 49)$ 


\section{L'IMMINENCE DE LA FIN}

Une idée courante au XviI ${ }^{e}$ siècle voulait que la civilisation se déplace de l'est vers l'ouest. Née en Asie, continuant sa course en Europe, la culture humaine trouvait logiquement son aboutissement (son apothéose et sa fin) en Amérique. «L'Amérique, dès son commencement, signifie la fin d'un monde ${ }^{15}$ ", écrit François Paré. La fin d'un monde qui, semble-t-il, ne finit plus d'advenir. Pour Unless et la narratrice de Pourquoi faire une maison avec ses morts, c'est au sein de leur univers immédiat que se réalisera l'apocalypse. Leur parcours prend la forme d'une attente douloureuse de cette fin. Évidemment, on pourrait soutenir que l'humanité est fascinée par l'idée de sa propre disparition depuis plus de 2000 ans ${ }^{16}$. Mais, dans ces textes, c'est du quotidien même que surgissent les signes de la fin, ceux-ci se manifestant avec une rare force à tout instant: «Le deuil était devenu si large, la menace si constante qu'il modifiait la signification du moindre événement d'une existence.» $(P, 72)$ Désastres intimes et collectifs sont placés sur le même pied, en ce qu'ils mettent en évidence un sujet en crise, oppressé par le sentiment d'un monde au bord de basculer.

Chez Hélène Monette domine l'impuissance du sujet devant les catastrophes qui se succèdent: «Nous n'y sommes pour rien, ni fonctionnaires, ni psychiatres, ni marchands d'armes.» $(U, 64)$ Sans pouvoir financier, politique ou médiatique, ces personnages « ordinaires » sont à la merci des événements, balancés d'un drame à l'autre avec la même intensité, jusqu'à ne plus pouvoir distinguer la source de leur mal:

Que les hôpitaux, les psychopathes, les ambulances, l'héroïne, la violence, [...] la Yougoslavie, les salles d'attente, le Rwanda, le Prozac, [...] mon frère, les seringues, ma mère, les mensonges, la drogue, la douleur, la Dette, la prostitution, les électrochocs et les anges m'avaient refroidie! Anéantie temporairement... $(U, 81)$

Tous les éléments sont ici posés sans hiérarchie, dans une fausse relation de proximité. Le portrait d'un monde décadent, qui ne se limite évidemment pas à l'Amérique, est malgré tout tracé à partir du sujet et du lieu qu'il occupe en Amérique du Nord, l'espace du corps étant assailli par les mots et les images de l'horreur, et la conscience se trouvant incapable de les mettre en forme. Si le pathétique est toujours gardé à distance par la mise en œuvre d'une poétique de l'exubérance, à mi-chemin entre l'ironie et le désespoir, la parole d'Unless se fait malgré elle prophétique: «Température fuckée due aux volcans, au ciel radioactif, à l'humanité tenue serrée. Les Amerloques refusaient toujours de signer le traité, le monde n'allait plus durer. Calvaire!» $(U, 105)$

$$
++
$$

15 François Paré, «Intérieurs et extérieurs de l'Amérique chez Pierre Nepveu», Voix et Images, vol. XXXIV, n 1 , automne 2008, p. 90.16 «Vivons-nous la fin des temps? La question nous revient sans cesse, depuis que le monothéisme et ses avatars ont enraciné la pensée du temps et de l'histoire dans l'angoisse de la rédemption et l'espoir d'un accomplissement. » Jean-François Chassay, Anne Élaine Cliche et Bertrand Gervais, "Présentation", Jean-François Chassay, Anne Élaine Cliche et Bertrand Gervais (dir.), Des fins et des temps. Les limites de l'imaginaire, Montréal, Université du Québec à Montréal et Figura, 2005, p. 7. 
Le recueil d'Élise Turcotte, quant à lui, culmine sur le début de la «vraie» fin du monde, à tout le moins sur une catastrophe naturelle de grande ampleur, évoquant le récit biblique du Déluge. Dans «Journal d'une mortelle», une pluie torrentielle s'abat sur la ville et les inondations chassent les habitants de leur maison. La narratrice et ses enfants, s'étant «abrités des jours et des jours au dernier étage d'un immeuble à bureaux » $(P, 103)$, finissent par réintégrer leur maison détériorée par l'eau. La narratrice participe aux mesures d'aide aux survivants en les guidant vers un hôpital psychiatrique : «Les blessés et les malades en occupent la majeure partie. Certains se sont jetés du toit des maisons les plus hautes. D'autres continuent seulement leur lutte contre la maladie.» $(P, 104) C^{\prime}$ est à l'intimité du désastre que nous avons accès et non pas à ses images les plus crues. La narratrice converse avec les gens dont elle prend soin, s'assure de combler leurs besoins; ses enfants adoptent des chats et des pigeons ; ils préparent les repas sur un réchaud de camping, s'absorbent dans des tâches dérisoires, à défaut de s'atteler à celles, plus colossales, qui les attendent. Un certain ordre se maintient malgré tout, et c'est peut-être ce qui est le plus effrayant. La vie après le Déluge ressemble étrangement à la vie d'avant.

Bien que les repères géographiques disséminés à travers le recueil nous laissent penser que nous sommes à Montréal, les paysages de ce dernier récit, avec ces maisons réduites à des boîtes de carton humides, évoquent de façon évidente La NouvelleOrléans après Katrina. Mais cette représentation d'un monde ravagé pourrait aussi se lire comme la radiographie de n'importe quelle agglomération nord-américaine. Sous l'éclatante peau des villes se trouvent des squelettes rongés, des fluides souillés, des organes contaminés. Et c'est de l'intuition de la catastrophe que les yeux reluisent, non de santé.

Traverser l'Amérique vers le dedans, tel que le proposait René Lapierre, prend ici la forme d'une quête mortifère, puisque le lieu occupé par le sujet se trouve constamment mis en péril. Chez Turcotte comme chez Monette, les narratrices sont animées par le désir d'un refuge, d'un endroit où échapper à la folie du monde et à la frénésie de l'Histoire, quête constamment mise en échec par l'étrangeté fondamentale du chezsoi. La représentation d'un espace social fragilisé, dont la décadence de la ville nordaméricaine constitue la figure principale, ainsi que celle d'un espace intime envahi par l'Autre, la maison et le corps apparaissant intoxiqués par la menace ambiante, nous permettent de réfléchir à nouveau sur la question de l'américanité. En effet, corps et continent, pris l'un pour l'autre, trahissent le même malaise et fondent d'une manière particulière la difficulté à habiter le monde. En parlant des États-Unis, Paul Virilio désignait leurs villes et leurs institutions comme "une prothèse jetée sur un continent ${ }^{17}$ ». Cette constatation s'appliquerait encore plus justement au Québec, où l'importance du contrôle exercé par la métropole (tant par la France que par l'Angleterre après la Conquête) a fortement influencé l'aménagement de l'espace tant

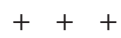

17 Paul Virilio, Essai sur l'insécurité du territoire, Paris, Stock, 1976, p. 31.

VOIX ET IMAGES $109 \quad 126$ 
physique que social, les villes et les institutions coloniales ayant d'abord été pensées en Europe. La formule de Virilio, en dehors de sa teneur fortement dénonciatrice, reste pertinente dans la mesure où elle propose une vision de l'Amérique où le sujet, depuis l'arrivée des Européens, serait foncièrement en rupture avec l'espace qu'il habite. L'image de la prothèse suggère un continent amputé d'une partie essentielle de son être remplacée temporairement par une architecture (sociale et urbaine) compensatoire ${ }^{18}$. De même, les œuvres de Monette et de Turcotte participent d'un imaginaire qui hante les fictions nord-américaines, celles-ci ne cessant de pointer ce hiatus infranchissable entre le sujet et son environnement, entre le sujet et son propre corps, ce dernier se révélant aussi étrange et douloureux que peut l'être un membre artificiel.

Benoît Doyon-Gosselin souligne qu'à partir des années 1980, «la difficulté d'habiter l'espace devient un leitmotiv des plus féconds autant dans le roman québécois que dans le roman d'expression française au Canada ${ }^{19}$ ». Il faudrait ajouter que ce motif est prégnant dans l'ensemble des littératures nord-américaines contemporaines, avec une importance particulière dans les fictions narratives écrites par des femmes (pensons à Louise Erdrich et à Lorrie Moore, aux États-Unis, ou à Alice Munro et à Barbara Gowdy, au Canada anglais). Le problème de l'habiter se situe au cœur de l'idée de l'Amérique, traversant les histoires littéraires de façon souterraine (Pierre Nepveu l'a bien montré) pour rejaillir avec force dans la deuxième moitié du Xxe siècle, à une époque où l'envers du rêve américain nous apparaît plus clairement que jamais.

La réflexion amorcée ici ne constitue que le point de départ d'un long travail qui reste à accomplir et qui consiste à évaluer comment ces écritures issues de l'Amérique, mais qui ne mettent pas à l'avant-plan l'Amérique comme thème, nous forcent malgré tout à redéfinir notre imaginaire du Nouveau Monde. Ce parti pris pour une autre forme d'américanité comporte un risque important, celui d'en faire un concept fourre-tout, ignorant les différences historiques et identitaires considérables entre les peuples d'Amérique du Nord et leur manière propre d'habiter le territoire. Pourtant, le but de ce travail serait plutôt de soulever une question ouvrant sur plusieurs réponses : comment fait-on l'expérience de l'Amérique aujourd'hui? Quels sont les éléments déterminants qui permettent de situer la littérature québécoise contemporaine dans le contexte nord-américain, dans son histoire et sa culture? D'où l'intérêt, ainsi que l'ont proposé Nepveu et, chacun à sa manière, Lapierre et Jean-François Chassay ${ }^{20}$, de poser l'américanité dans la perspective d'une expérience subjective à partir de laquelle appréhender le continent dans sa complexité et sa pluralité. Toujours, il n'est question que de comprendre comment la fiction travaille à rendre lisible ce

$+++$

18 À travers son analyse phénoménologique du motel, le philosophe Bruce Bégout nous en donne un exemple probant: «Emblème de l'instabilité chronique de tout un pays, le motel représente la forme d'installation typique d'une ville qui n’a pas su s'ancrer définitivement dans le sol, mais se complaît dans les abris temporaires. [...] En dépit de leur ancienneté et de leur solidité relatives, toutes les constructions architecturales de la ville américaine possèdent cet aspect précaire et accidentel.» Bruce Bégout, Lieu commun. Le motel américain, Paris, Allia, 2003, p. 41. 19 Benoît Doyon-Gosselin, «Les figures spatiales dans L'̂̂le de la Merci d'Élise Turcotte ou la maison de l'emprisonnement", Voix et Images, vol. XXXII, n 3, printemps 2007, p. 123.20 Jean-François Chassay, L'ambiguité américaine. Le roman québécois face aux États-Unis, Montréal, XYZ éditeur, coll. «Théorie et littérature», 1995, $197 \mathrm{p}$. 
continent, dont la découverte marquait la possibilité de fonder un homme nouveau sur une terre nouvelle, et dont les écrivains ne cessent de raconter le désenchantement qui résonne encore quatre siècles plus tard. 